\title{
Radionuclide Production for the Biosciences
}

Thomas J. Ruth

UBC/TRIUMF PET Program

\section{INTRODUCTION}

The use of radionuclides in the bioscience can be broken down into three general categories, imaging, radiotherapy and radiotracers. Imaging can be further divided into positron emission tomography (PET) and single photon emission tomography (SPECT). These topics are beyond the scope of this paper and will not be described here.

All of these uses rely on the fact that the radionuclides are used at tracer concentration. In order to be used as tracers the radionuclides and the compounds to which they are attached must obey the tracer principles which state that:

- the tracer behaves or interacts with the system to be probed in a known, reproducible fashion,

- tracer does not alter or perturb the system in any fashion and

- the tracer concentration can be measured.

In radiotherapy, the second principle is, in a strict sense, broken since the point of delivering the radiotoxic substance is to have the emitted radiation cause damage to the undesirable surrounding tissues. However, in order for the radiotoxic substance to localize it must be follow the known chemical behaviour without perturbing that pathway.

The following are some typical radionuclides used in each of the broad categories:

${ }^{11} \mathrm{C}$ is a positron emitting radionuclide with a half-life of 20 minutes. It is generally produced as ${ }^{11} \mathrm{CO}_{2}$ which can be converted into a wide variety of labelling agents such as ${ }^{11} \mathrm{CH}_{3} \mathrm{I}$ or $\mathrm{H}^{11} \mathrm{CN}$. Since all biological compounds contain carbon ${ }^{11} \mathrm{C}$ finds wide spread use as a tracer in PET.

${ }^{13} \mathrm{~N}$ is also a positron emitting radionuclide. However it is used in applications other than PET imaging, for example it is widely used in botany studies to determine the kinetics of nitrogen uptake in a variety of plant systems.

${ }^{123} \mathrm{I}$ emits -rays with an energy of $159 \mathrm{keV}$ which is ideally suited with imaging in SPECT cameras. In addition the ease with which an iodine atom can be inserted into a compound makes ${ }^{123} 1$ extremely versatile as a radiotracer in SPECT.

\footnotetext{
${ }^{186} \mathrm{Re}$ is a $\beta$ - emitter with a low abundant $\gamma$-ray with an energy of $140 \mathrm{keV}$. The $1 \mathrm{MeV} \gamma$-rays and its 90 hour half-life make it a promising radiotoxic nuclide for therapy. As a chemical analogue of technetium, thenium possesses similar chemical properties as ${ }^{9 \%} \mathrm{Tc}$ and can thus be used with some of the same compounds that have been developed for imaging tumours.
}

Most of the radiotracers have relatively short half-lives (less than a few hours to at most a few days). There are definite advantages in using short-lived radionuclides, for example there is a low radiation dose associated with each study, serial studies are possible (sometimes on the same day for tracers such as ${ }^{11} \mathrm{C}$ ) and the radioactive waste disposal problems are minimized if not eliminated. The disadvantages include the need for an accelerator nearby or within easy shipping distance for the longer lived species and rapid chemical procedures are required, especially for the more complex compound formation.

\section{RADIONUCLIDE PRODUCTION}

The rate of production is dependent on the number of bombarding particles, the number of target nuclei and the cross section of the reaction. The length of bombardment is also important since the production of short lived nuclei can reach saturation rather quickly. In order to optimize the production of a particular radionuclide the following production considerations must be taken into account:

- the threshold energy for the desired reaction,

- the energy where the maximum yield occurs,

- the chemical form of the target nucleus,

- the physical form of the target nucleus,

- the chemical form of the desired product,

- the physical form of the desired product, and

- the ease of separation of product from the target.

To illustrate this approach we can look at the case where ${ }^{18} \mathrm{~F}$ is needed as fluoride. The two most widely used nuclear reactions for producing ${ }^{18} \mathrm{~F}$ are the ${ }^{20} \mathrm{Ne}(\mathrm{p}, \alpha){ }^{18} \mathrm{~F}$ and the ${ }^{18} \mathrm{O}(\mathrm{p}, \mathrm{n})^{18} \mathrm{~F}$. Both reactions have a relatively low threshold energy $(0-2 \mathrm{MeV})$ and the maximum yield occurs under 10 $\mathrm{MeV}$. However, the $(p, n)$ reaction has a much higher cross section so that the quantity of ${ }^{18} \mathrm{~F}$ that can be produced expressed as $\mathrm{mCi} / \mu \mathrm{Ah}$ is more than double for a thick target reaction using protons of about $12 \mathrm{MeV}$ in comparison to deuterons of comparable energy [1]. Thus having selected the mode of production and the energy, the next issue is to examine the chemical and physical forms of the target and product. The most convenient target material for producing "F as fluoride is a water target enriched in ${ }^{18} \mathrm{O}$. The resulting ${ }^{18} \mathrm{~F}$ is produced directly as the fluoride ion which can be extracted from the target water by simple ion exchange columns.

\section{ACCELERATORS}

Through the 1970's most radionuclides that were produced for the biomedical community utilized cyclotrons designed for physics applications. These machines usually accelerated four 
particles and were cumbersome to operate when it came to changing particles which was necessary since the preferred reactions for the PET radionuclides required both protons and deuterons (e.g. ${ }^{14} \mathrm{~N}(p,)^{11} \mathrm{C}$ and ${ }^{14} \mathrm{~N}(\mathrm{~d}, \mathrm{n}){ }^{15} \mathrm{O}$ ). In the late 1970 's the cross sections for proton induced reactions for the PET radionuclides ${ }^{18} \mathrm{~F}$ and ${ }^{15} \mathrm{O}$ were explored and found to give higher yields than the corresponding deuteron reactions. These results and the fact that all of the commercially available radionuclides produced for nuclear medicine are also produced by proton induced reactions led the participants of the Heidelberg Workshop on Targetry and Target Chemistry to conclude that proton only cyclotrons could meet the needs of the PET/nuclear medicine communities [2]. The primary drawback to this possession was the fact that some of the reactions required the use of enriched target materials which were not always readily available or easily recovered. More recently the availability issues have been resolved for the most part.

Another factor that has influenced the expansion of PET was the development of negative ion cyclotrons which were made possible by the demonstration of the principle of accelerating negative hydrogen ions and extracting the protons by electron stripping [3]. The first negative ion cyclotron was built at UCLA and the University of Manitoba in the mid 1960's [4,5]. TRIUMF, Canada's National Laboratory for Nuclear and Particle Physics was established around the design of a 500 $\mathrm{MeV}$ negative ion cyclotron [6]. By the late 1970's The Cyclotron Corporation (TCC) had designed and built several negative ion cyclotrons for the producing the commercial radioisotopes and for neutron radiotherapy (CP42 and CP45) [7]. After CTl took over TCC's holdings they built a small negative ion cyclotron producing protons of $11 \mathrm{MeV}$ [8]

The advantages of a negative ion machine include the simplicity with which the protons can be extracted, the near quantitative extraction efficiency, the ability to vary the extraction energy easily and the ability to extract multiple beams simultaneously.

At the same time these events were occurring the development of external ion sources added another advantage to the negative ion machines. These new ion sources produced very high beam currents with low emittance, the centre region is not disturbed with replacement of the ion source, the vacuum tank does not have to opened during repairs of the ion source and high vacuum is more easily maintained with an external ion source which is differentially pumped.

\section{A. How Big?}

In selecting an accelerator for producing radionuclides for biomedical research the hardest question to answer is how big an accelerator is needed? The real question is what energy since the smaller the machine the less valuable space is required. The obvious answer to this question is in the definition of the program involved. For example, a program to produce the commercial radioisotopes such as ${ }^{123} \mathrm{I}$ and ${ }^{201} \mathrm{Tl}$ requires a machine capable of providing protons of about $30 \mathrm{MeV}$ while a clinical PET program can have its needs met with an accelerator with 10-13 MeV protons. PET research programs may a higher energy accelerator.

\section{B. Alternatives.}

Along with the research into using high beam power targets came a renewed interest in low energy alternatives. Several different approaches were examined. One proposed using a low energy tandem cascade accelerator to generate protons and deuterons of 3-4 MeV [9]. While the cross sections for the various reactions are low at these energies the idea was to make up the low production rate with higher beam current. The use of deuterons of 3-4 MeV has proven useful not only for the tandem cascade accelerator but also in a small dedicated $3 \mathrm{MeV}$ cyclotron used just to make ${ }^{15} \mathrm{O}$-labelled compounds. Another approach proposed using ${ }^{3} \mathrm{He}$ as the accelerated particle. The advantage to this machine would be the low neutron flux associated with the reactions [10].

In addition to these new machines an interest in linear accelerators has also been generated [11]. The major advantage with these machines would be the low cost, low weight and low radiation generated by the machine, thus they would require less shielding around the machine itself. Regardless of the source of the accelerated particles the targets would be similar except for the very low energies where new concepts have had to be explored due to the large energy loss as the projectiles pass through matter (e.g. isolation foils) at the low energies.

\section{Yields}

The actual yields from most target systems is generally less than the theoretical predictions. This is especially true for gas target systems. Possible reasons for low yield at high beam currents in gas targets could be the interaction of the produced radionuclide with the target chamber walls, gas density reduction due to beam heating of the gaseous target material and thus the lack of optimization of the energy of the projectile. It has been clearly demonstrated that gas density reduction is a major contributor to loss of yield as a function of beam current.

\section{FUTURE}

The funure growth area for nuclear medicine is predicted to be in radiotherapy. While most radiotoxic nuclides emit particles and are thus neutron rich and are generally produced in a reactor, there is keen interest in producing these radiotoxic nuclides via particle accelerators because of the inherent ability to produce a radionuclide of differing chemical element with accelerators, thus increasing the potential specific activity. 


\section{A. NBTF.}

No discussion of the production of radionuclides for the biosciences can be complete without some mention of the proposed National Biomedical Tracer Facility, the NBTF. The nuclear medicine community in the US has seen a need for a dedicated accelerator facility that would be capable of produced a wide range of radionuclides for research proposes. Presently these radionuclides are prepared at the national labs but this approach has proven to unsatisfactory due to limited operated schedules and a lack of control of what is produced. The commercial suppliers are not interested in supplying radionuclides that have low or no profit margin. Thus new promising what to radiotherapy are being stymied because of the lack of availability of some radionuclides. The proposed NBTF would conceivably go a long way toward relieving this problem. The proposal is to build an accelerator cable of generating at least two beams of protons of up to $500 \mu \mathrm{A}$ each with variable energies up to $80 \mathrm{MeV}$. The machine would be available throughout the year operating 24 hours per day, 7 days per week for a minimum of 48 weeks. In addition to the production of radionuclides, the mission of the NBTF would include education and training in nuclear and radiochemistry, research in target and target chemistry and research in the use of new radiopharmaceuticals. Having been reviewed for the Department of Energy (DOE) by the National Academy of Sciences' Institute of Medicine, the DOE is reviewing a number of Project Definition Studies from a number of sites throughout the US [12]. It is still not clear whether the resources to build a new facility will be made available.

\section{ACKNOWLEDGEMENTS}

The author wishes to acknowledge the helpful comments and efforts of colleagues at TRUUMF, especially Drs. Michael Adam and Stefan Zeisler and Salma Jivan, Tamara Hurtado and Ken Buckley. Support from TRIUMF and the Medical Research Council of Canada is also gratefully acknowledged.

\section{REFERENCES}

[1] Ruth, TJ, Wolf AP, Radiochim. Acta (1979); 26: 21-24.

[2] Proc. 1st Workshop on Targetry and Target Chemistry, Heidelberg (1985). 18-19.

[3] Rickey ME, Smythe R, Nucl. Instr. Meth, (19620; 66

[4] Wright BT, IEEE Trans. (1966); NS-13: 72.

[5] Burgerjon JJ, Nucl. Instr. Meth. (1966); $43: 381$

[6] Burgerjon JJ, Nucl. Instr. Meth. (1985); B10/11: 951956.
[7] Hendry GO, Kuo TYT, Ramsey F, Jenkins K, et al. 9th Int. Conf. Cyclotrons and their Appl. Les eds. de Physique, Paris (1982): 125 .

[8] Wieland BW, Hendry GO, Schmidt DG, J. Label. Cmpds. Radiopharm. (1985); 23: 1187.

[9] Shefer RE, Klinkowstein RE, Welch MJ, Brodack JW, Proc. 3rd Workshop on Targetry and Target Chemistry, Vancouver, (1990).

[10] Dabri AE, Hagan WK, Swenson DA, Krohn KA, Proc. 3rd Workshop on Targetry and Target Chemistry, Vancouver, (1990)

[11] Hamm RW, Proc. 3rd Workshop on Targetry and Target Chemistry, Vancouver, (1990).

[12] Isotopes for Medicine and the Life Sciences, SJ Adelstein and FJ Manning, eds. Nat. Acad. Sci. Press (1995). 
